was undertaken to review the evidence of interactions between cannabis-based medicinal products and other ASMs.

Methods A search of Cochrane, Pubmed and Embase from January 2015 to April 2020 was performed. All original research papers investigating interactions between cannabidiol and ASMs were included. Bibliographies of review articles were searched to identify further papers. Adverse events and side effects were excluded.

Results Thirty studies (eighteen observational cohort studies, two randomised-control trials, three case reports/series, three animal studies, two briefing reports, an analysis of cohort data and a clinical trial simulation) were identified. Pharmacokinetic interactions were identified between $\mathrm{CBD}$ and clobazam, rufinamide, zonisamide, stiripentol, sirolimus/everolimus, eslicarbazepine, brivaracetam, potassium bromide, tiagabine and gabapentin. Pharmacodynamic interactions were identified for clobazam, valproate and levetiracetam. There is further contention regarding whether pharmacokinetic interactions are present with topiramate, oxcarbazepine, lacosamide, phenobarbital and pregabalin. An animal study identified that the brain concentration of ASMs may alter while the serum concentration remains the same.

Conclusions The studies to date suggest a number of possible interactions between CBD and ASMs. Possible mechanisms include alterations in metabolism through cytochrome p450. The existing literature is limited for some ASMs, with studies having relatively small cohorts. As increasing numbers of patients use cannabidiol, clinicians need to monitor closely for interactions clinically and with blood levels when needed.

\section{G52(P) TYPE I CHIARI MALFORMATION IN TWO SIBLINGS: SYNCOPE, THE PRESENTING FEATURE}

${ }^{1}$ MBKC Dayasiri, 'S Kodagali, 'N Weerapperuma, 'E Goh, ${ }^{2} S$ Magdum, ' $G$ Anand. ${ }^{1}$ Paediatrics, Oxford University Hospital, Oxford, UK; ${ }^{2}$ Paediatric Neurosurgery, Oxford University Hospital, Oxford, UK

\subsection{6/archdischild-2020-rcpch.38}

Introduction Type I Chiari malformation is descent of the cerebellar tonsils into the cervical canal and is usually not associated with hydrocephalus. Although many affected children are asymptomatic, presenting features can include headaches, ocular disturbances, otoneurologic disturbances, lower cranial nerve signs, cerebellar ataxia, or spasticity. Chiari drop attacks/ syncope as a presentation is not widely recognised and through this report we attempt to highlight this feature.

Case Report The reported children were born to consanguineous parents yet with an uncomplicated antenatal period. Both children had age appropriate development. The elder sibling presented at 11 years of age with repeated episodes of collapse. She had headache for the preceding three years. There was no history of paraesthesia, blurred vision, tinnitus, vomiting, clumsiness, neck pain or any shooting pains down her back and legs. Neurological examination was normal. MRI Brain revealed $8 \mathrm{~mm}$ herniation of the cerebellar tonsils through the foramen magnum consistent with a Chiari 1 malformation. There was no evidence of cervical and upper transthoracic syrinx or hydrocephalus. She was managed conservatively with regular follow up.

The younger sibling first presented at 2 years of age repeated episodes of drowsiness and sweating as the first event in morning which was attributed to ketotic hypoglycaemia. However, she presented at 6 years of age with a syncopal episode lasting for a minute which happened as she was jumping on a bed. Neurological examination was normal. MRI brain revealed Chari I malformation with significant tonsillar herniation (more than $1.6 \mathrm{~cm}$ up to the $\mathrm{C} 2$ vertebra in the neck) which was causing significant congestion at the foramen magnum needing urgent neurosurgical decompression.

Conclusion This report highlights importance thorough evaluation of syncope/drop attacks in children as it could be rarely a presenting feature of Chari 1 malformation. The aetiology of Chari I malformation in these children is likely genetic with autosomal recessive inherence given the parents were consanguineous and both children were affected.

\section{G53(P) PAROXYSMAL KINESIGENIC DYSKINESIA; A DIAGNOSTIC CHALLENGE TO THE GENERAL PAEDIATRICIAN}

MBKC Dayasiri, N Weerapperuma, J Wright, G Anand. Department of Paediatrics, Oxford University Hospital, Oxford, UK

\subsection{6/archdischild-2020-rcpch.39}

A ten-year old girl presented with a month's history episodic facial and limb movements. She had a normal neurological examination and based on video evidence, she was thought to have complex tics. Anxiety was reported as being a trigger. Unusually, these 'tics' were not directly witnessed during hospital visits. Eighteen months after initial presentation, the clinician subsequently observed dystonic posturing after the child stood up from having been seated in a chair. Paroxysmal kinesigenic dyskinesia (PKD) was then suspected and confirmed with genetic testing. She was successfully treated with carbamazepine. Retrospectively, it was evident that her anxiety was related to a fear of uncontrolled movements, rather than it being a trigger.

The abnormal involuntary movements in PKD are precipitated by sudden voluntary movement. Lack of recognition of this typical feature, normal examination, and/or features such as anxiety can lead to misdiagnosis or delayed diagnosis of this easily treatable condition.

\section{G54(P) DISABILITY AND VISUAL OUTCOMES FOLLOWING ABUSIVE HEAD TRAUMA (AHT) IN CHILDREN UNDER 2 YEARS}

1) Wright, ${ }^{2} \mathrm{~S}$ Painter, 'S Kodagali, ${ }^{1} \mathrm{~N}$ Jones, 'S Jayawant, ${ }^{2} \mathrm{~J}$ Elston, ${ }^{1} \mathrm{G}$ Anand. ${ }^{1}$ Department of Paediatrics, Oxford University Hospitals NHS Trust, Oxford, UK; ${ }^{2}$ Department of Ophthalmology, Oxford University Hospitals NHS Trust, Oxford, UK

\subsection{6/archdischild-2020-rcpch.40}

Aim The primary objective was to report immediate and longterm disability outcomes in children following an abusive head trauma (AHT) aged less than 2 years. The secondary objective was to report visual outcomes.

Methods A retrosepctive study that identified children from the Ophthalmology Department's database of children examined for suspected AHT, between 1995 and 2017. Children were included if they were aged 24 months or less, with no pre-existing visual or developmental problems, and if their injury was confirmed as a result of AHT. This was determined by the conclusion of the local Multi-Disciplinary Team. Ethical approval was obtained. 